\title{
A Step-Up/Step-Down Switched-Capacitor AC-AC Converter with Symmetrical Topology
}

\author{
Kei Eguchi ${ }^{1}$, Kanji $\mathrm{Abe}^{2}$, Ichirou Oota ${ }^{3}$, and Hirofumi Sasaki ${ }^{4}$ \\ ${ }^{1,2}$ Department of Information Electronics, Fukuoka Institute of Technology, \\ 3-30-1 Wajirohigashi, Higashi-ku, Fukuoka, Japan \\ ${ }^{3}$ Department of Information, Communication and Electronic Engineering, National Institute of Technology, \\ Kumamoto College, 2659-2 Suya, Koushi, Kumamoto, 861-1102 Japan \\ ${ }^{4}$ Tokai University, 9-1-1 Toroku, Kumamoto Japan
}

\begin{abstract}
In this paper, a new step-up/step-down AC-AC converter is designed by switched capacitor (SC) techniques. The main features of the proposed SC AC-AC converter are the symmetrical converter topology and the absence of magnetic elements. Owing to the symmetrical converter topology, the number of capacitors in the proposed AC-AC converter is fewer than that in the conventional SC AC-AC converter. Furthermore, the proposed AC-AC converter has a smaller SC resistance than the conventional SC AC-AC converter. Therefore, the proposed $A C-A C$ converter can improve power efficiency and input power factor from the conventional $S C$ $A C-A C$ converters. The principle of operation, qualitative analysis, and simulation evaluation are described concerning the proposed AC-AC converter with three stages. The results of the simulation program with integrated circuit emphasis (SPICE) simulation demonstrate the feasibility and effectiveness of the proposed ACAC converter.
\end{abstract}

Keywords: AC-AC converters, switching converters, switched capacitor techniques, step-up/step-down converters, symmetrical topology

\section{Introduction}

An autotransformer is one of the most popular solutions to provide stepped-up or stepped-down voltages. Unlike a voltage transformer which has two isolated windings, the autotransformer has the usual magnetic core but only has one winding. However, like any transformer, the autotransformer is difficult to realize high power efficiency. Furthermore, due to the magnetic core and winding, the autotransformer is heavy and bulky. To overcome these problems, several types of AC-AC converters have been designed by using switched capacitor (SC) techniques [1]-[7]. As the SC AC-AC converters require no magnetic components, light weight and small converter size can be realized by the SC AC-AC converters.

As far as the authors know, the first SC AC-AC converter was suggested by Ueno et al. in 1993 [1]. By changing the connection of $N(=2,3, \ldots)$ charge-transfer capacitors by $N$-phase clock pulses, Ueno's AC-AC converter offers a staircase AC waveform [1]. Following this study, Terada et al. [2] and Eguchi et al. [3] proposed a ring-type SC AC-AC converter in order to enhance the flexibility of conversion ratios. Being distinct from such purpose, a simple SC AC-AC converter was suggested by Lazzarin et al. [4],[5] and Andersen et al. [6]. By controlling bidirectional switches by two-phase clock pulses, the conventional converters reported in [4][6] offer 1/2x stepped-up and 2x stepped-up voltages. Following this study, You et al. [7] expanded Lazzarin's AC-AC converter to realize the conversion ratio of 1/4. These SC AC-AC converters reported in [4]-[7] have simple converter topology though the conversion ratio is limited. However, there is still room for improvement in the converter topology of the conventional SC AC-AC converters.

This paper proposes a new step-up/step-down AC-AC converter designed by using switched capacitor (SC) techniques. Unlike the conventional SC AC-AC converters reported in [4]-[7], the proposed AC-AC converter has symmetrical converter topology. Owing to the symmetrical converter topology, the proposed AC-AC converter achieves not only fewer capacitors but also a smaller SC resistance than the conventional SC AC-AC 
converters. Therefore, the proposed AC-AC converter can improve power efficiency and input power factor from the conventional SC AC-AC converters. To confirm the validity of the proposed converter, simulation program with integrated circuit emphasis (SPICE) simulations and theoretical analysis are performed.

The rest of this paper is organized as follows. In section 2, the circuit configuration of the proposed AC-AC converter is presented. In section 3, the property of the proposed AC-AC converter is analyzed by assuming a four-terminal equivalent circuit. Simulation results are shown in Section 4. Finally, conclusion and future work are drawn in section 5 .

\section{Circuit Configuration}

\subsection{Conventional AC-AC converter}

Figure 1 shows the conventional switched-capacitor (SC) AC-AC converter suggested by Andersen et al [6]. The converter topology of figure 1 is based on the SC DC-DC converter proposed by Hara et al [8]. As figure 1 shows, the conventional AC-AC converter consists of four transistor switches and three capacitors, where bidirectional switches $S_{1}$ and $S_{2}$ are driven by non-overlapped two phase clock pulses. By controlling these switches, the conventional converter realizes the following conversion ratios:

$$
\frac{v_{\text {out }}}{v_{\text {in }}}=\frac{1}{2} \quad \text { and } \quad \frac{v_{\text {out }}}{v_{\text {in }}}=2 \text {. }
$$

Of course, as reported in [7], the conventional converter can achieve the $N(=2,3,4, \ldots)$ x step-up and $1 / N(=2,3$, $4, \ldots)$ x step-down conversion. However, there is still room for improvement in the converter topology.
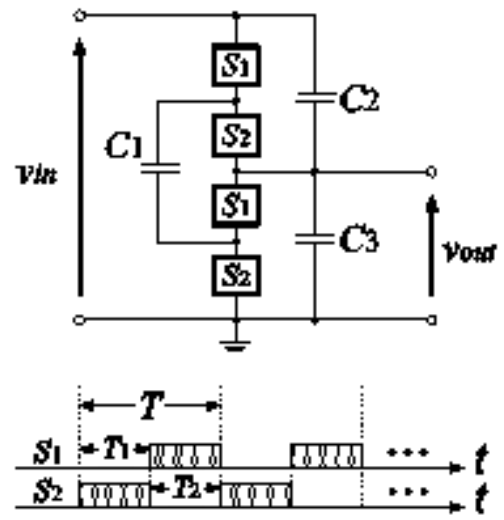

(a)
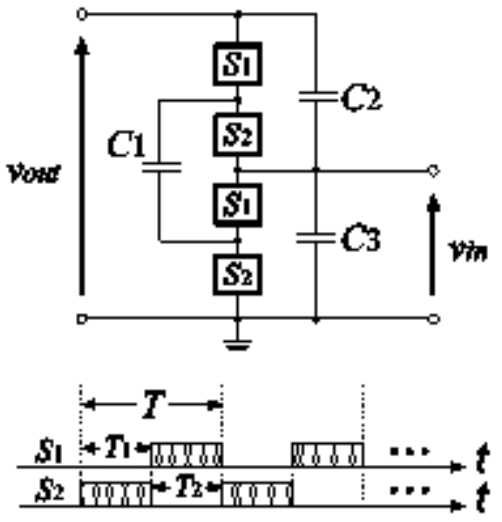

(b)

Fig. 1: Conventional SC AC-AC converter: (a) Step-down mode and (b) Step-up mode.

\subsection{Proposed AC-AC converter}

Figure 2 shows the proposed SC AC-AC converter with one stage. The proposed AC-AC converter consists of eight bidirectional switches and two capacitors. By controlling bidirectional switches, the proposed converter realizes the following conversion ratios:

$$
\frac{v_{\text {out }}}{v_{\text {in }}}=\frac{1}{2} \quad \text { and } \quad \frac{v_{\text {out }}}{v_{\text {in }}}=2 .
$$

As figure 2 shows, the proposed converter has symmetrical converter topology. For this reason, unlike the conventional converter, the proposed AC-AC converter can achieve not only fewer capacitors but also a smaller SC resistance than the conventional SC AC-AC converters. Of course, by increasing the number of stages, the proposed AC-AC converter can enhance flexible conversion ratios. Figure 3 shows the proposed converter with three stages. The proposed converter of figure 3 can realize the following conversion ratios:

$$
\frac{v_{\text {out }}}{v_{\text {in }}}=\left\{\frac{1}{4}, \frac{1}{3}, \frac{1}{2}, \frac{2}{3}, \frac{3}{4}, \frac{3}{2}, 2,3,4\right\} \text {. }
$$


For example, the proposed converter offers a $1 / 4 \mathrm{x}$ stepped-down voltage when the input terminal is $v_{i n}$ and the output terminal is $v_{\text {out }}$. As (3) shows, the conversion ratio of figure 3 is the same as that of the conventional converter reported in [7].

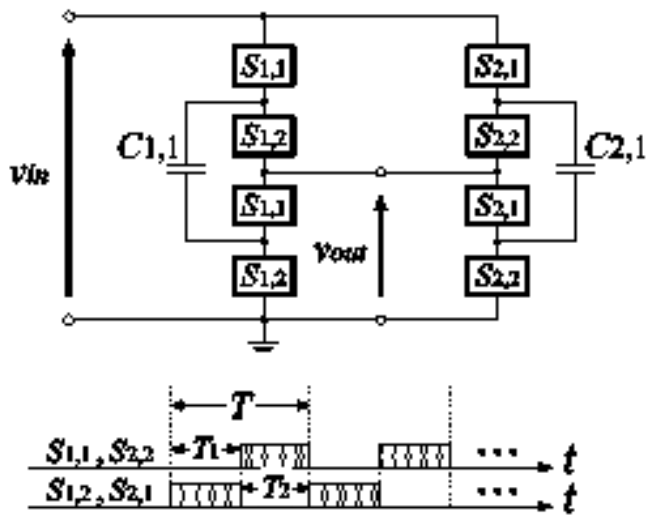

(a)

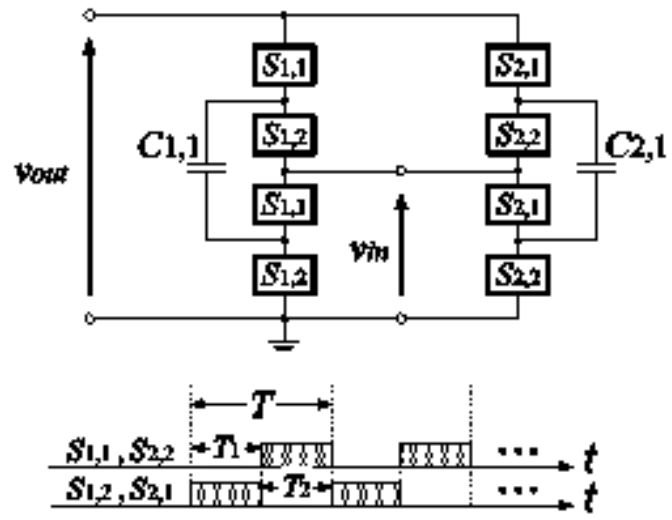

(b)

Fig. 2: Proposed SC AC-AC converter with one stage: (a) Step-down mode and (b) Step-up mode.

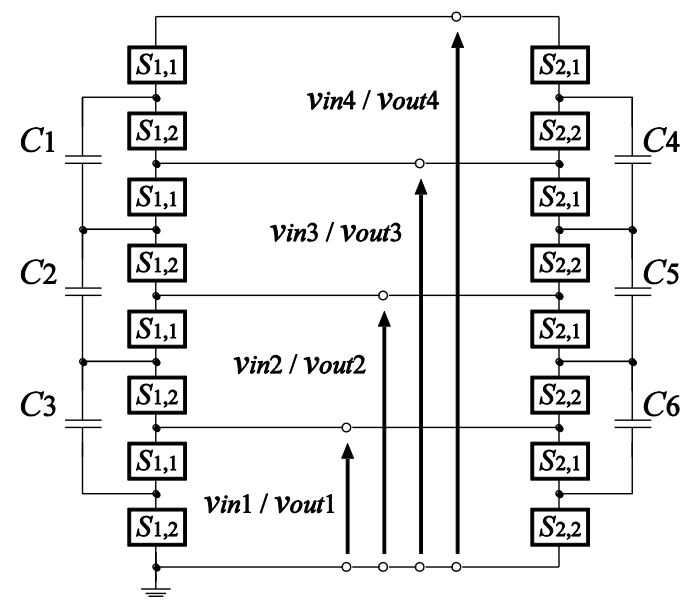

Fig. 3: Proposed SC AC-AC converter with three stages.

\section{Theoretical Analysis}

As an example, the characteristics of the proposed $\mathrm{AC}-\mathrm{AC}$ converter are analyzed in the conversion ratio of $1 / 4$ theoretically. In the theoretical analysis, the AC input is assumed as a staircase AC waveform in order to estimate the maximum power efficiency and the maximum output voltage. For the staircase AC waveform, the proposed AC-AC converter behaves like a DC-DC converter. Therefore, we analyse the proposed AC-AC converter by using a four-terminal equivalent circuit shown in figure 4, because it is known that an SC DC-DC converter can be expressed by a K-matrix [9]. In figure $4, R_{s c}$ is called the SC resistance and $m_{1}$ denotes the conversion ratio of an ideal transformer. In the theoretical analysis, these parameters are derived by using instantaneous equivalent circuits.

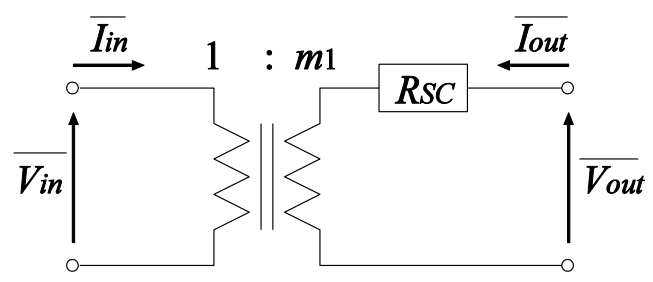

Fig. 4: Four-terminal equivalent circuit. 


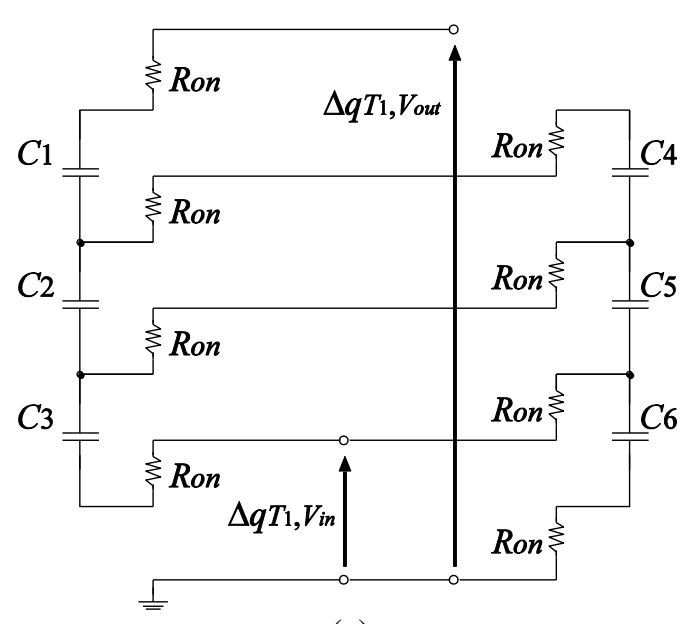

(a)

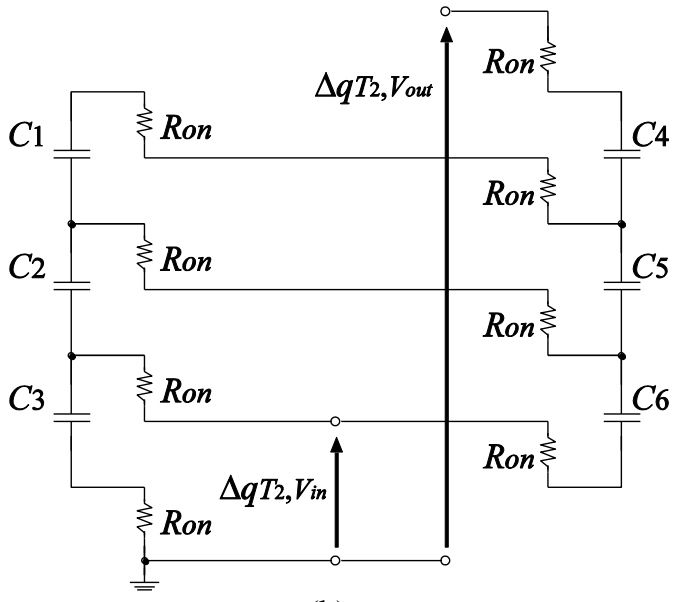

(b)

Fig. 5: Instantaneous equivalent circuits in the conversion ratio of 1/4: (a) State- $T_{1}$ and (b) State- $T_{2}$.

Figure 5 shows the instantaneous equivalent circuits of figure 3 in the conversion ratio of 1/4. In figure $5, R_{o n}$ denotes the on-resistance of switches. In the steady state, the differential value of electric charges in $C_{k}(k=1,2$, $\ldots, 6)$ satisfies the following equation:

$$
\Delta q_{T_{1}}^{k}+\Delta q_{T_{2}}^{k}=0
$$

where $\Delta q_{T i}{ }^{k}((i=1,2)$ and $(k=1,2, \ldots, 6))$ denotes the electric charge of the $k$-th capacitor in State- $T_{i}$. The interval of State- $T_{i}$ satisfies the following conditions:

$$
T=T_{1}+T_{2} \quad \text { and } \quad T_{1}=T_{2}=\frac{T}{2}
$$

where $T$ is a period of the clock pulse and $T_{i}(i=1,2)$ is the interval of State- $T_{i}$.

In State- $T_{1}$, the differential values of electric charges in the input and the output, $\Delta q_{T 1, v i n}$ and $\Delta q_{T 1, v o u t}$, are obtained as

$$
\begin{array}{ll} 
& \Delta q_{T_{1}, v_{\text {in }}}=\Delta q_{T_{1}}^{1}, \\
& \Delta q_{T_{1}, v_{\text {out }}}=-\Delta q_{T_{1}}^{3}-\Delta q_{T_{1}}^{5}+\Delta q_{T_{1}}^{6}, \\
& \Delta q_{T_{1}}^{1}=\Delta q_{T_{1}}^{2}+\Delta q_{T_{1}}^{4}, \\
& \Delta q_{T_{1}}^{2}=\Delta q_{T_{1}}^{3}-\Delta q_{T_{1}}^{4}+\Delta q_{T_{1}}^{5} .
\end{array}
$$

On the other hand, in State- $T_{2}$, the differential values of electric charges in the input and the output, $\Delta q_{T 2, v i n}$ and $\Delta q_{T 2, v o u t}$, are obtained as

$$
\begin{array}{ll} 
& \Delta q_{T_{2}, v_{\text {it }}}=\Delta q_{T_{2}}^{4}, \\
& \Delta q_{T_{2}, v_{\text {out }}}=-\Delta q_{T_{2}}^{2}+\Delta q_{T_{2}}^{3}-q_{T_{2}}^{6}, \\
& \Delta q_{T_{2}}^{4}=\Delta q_{T_{2}}^{1}+\Delta q_{T_{2}}^{5}, \\
& \Delta q_{T_{2}}^{5}=-\Delta q_{T_{2}}^{1}+\Delta q_{T_{2}}^{2}+\Delta q_{T_{2}}^{6} .
\end{array}
$$

Using (6) and (7), the average input current and the average output current can be expressed as

and

$$
\begin{aligned}
& i_{\text {in }}=\frac{\Delta q_{v_{\text {in }}}}{T}=\frac{\Delta q_{T_{1}, v_{\text {in }}}+\Delta q_{T_{2}, v_{\text {in }}}}{T} \\
& \overline{i_{\text {out }}}=\frac{\Delta q_{v_{\text {out }}}}{T}=\frac{\Delta q_{T_{1}, v_{\text {out }}}+\Delta q_{T_{2}, v_{\text {out }}}}{T} .
\end{aligned}
$$

In (8), $\Delta q_{\text {vin }}$ and $\Delta q_{\text {vout }}$ are electric charges in $v_{\text {in }}$ and $v_{\text {out }}$, respectively. Substituting (4)-(7) into (8), we have the relation between the input current and the output current as follows:

$$
i_{\text {in }}=-\frac{1}{4} i_{\text {out }} \text {. }
$$

From (9), the conversion ratio in figure 4 is obtained as $m_{1}=1 / 4$. 
Next, in order to derive the SC resistance $R_{S C}$, the consumed energy in one period is discussed. From figure 5, the consumed energy $W_{T}$ in one period can be expressed as

$$
W_{T}=W_{T_{1}}+W_{T_{2}}=2 W_{T_{1}},
$$

where

$$
W_{T_{1}}=\frac{\left(\Delta q_{T_{1}}^{1}\right)^{2}}{T_{1}} R_{o n}+\frac{\left(\Delta q_{T_{1}}^{4}\right)^{2}}{T_{1}} 2 R_{o n}+\frac{\left(\Delta q_{T_{1}}^{2}-\Delta q_{T_{1}}^{3}\right)^{2}}{T_{1}} 2 R_{o n}+\frac{\left(\Delta q_{T_{1}}^{3}\right)^{2}}{T_{1}} R_{o n}+\frac{\left(\Delta q_{T_{1}}^{6}\right)^{2}}{T_{1}} R_{o n}+\frac{\left(\Delta q_{T_{1}}^{5}-\Delta q_{T_{1}}^{6}\right)^{2}}{T_{1}} R_{o n} .
$$

Using (4)-(7), the consumed energy (10) is rewritten as

$$
W_{T}=\frac{3\left(\Delta q_{\text {out }}\right)^{2}}{2 T} R_{\text {on }} \text {. }
$$

Here, the consumed energy $W_{T}$ of the four-terminal equivalent circuit shown in figure 4 is obtained as

$$
W_{T}=R_{S C} \frac{\left(\Delta q_{\text {out }}\right)^{2}}{T} \text {. }
$$

Therefore, from (11) and (12), we have the SC resistances as follows:

$$
R_{S C}=\frac{3}{2} R_{o n} \text {. }
$$

By combining (9) and (13), the parameters in figure 4 are obtained as $m_{1}=(1 / 4)$ and $R_{S C}=(3 / 2) R_{o n}$. Therefore, the equivalent circuit of the proposed $\mathrm{AC}-\mathrm{AC}$ converter can be expressed by the following determinant:

$$
\left[\begin{array}{c}
v_{\text {in }} \\
i_{\text {in }}
\end{array}\right]=\left[\begin{array}{cc}
4 & 0 \\
0 & 1 / 4
\end{array}\right]\left[\begin{array}{cc}
1 & (3 / 2) R_{\text {on }} \\
0 & 1
\end{array}\right]\left[\begin{array}{c}
v_{\text {out }} \\
-i_{\text {out }}
\end{array}\right] .
$$

From (14), the maximum efficiency and the maximum output voltage can be derived as follows:

$$
\begin{array}{ll} 
& \eta_{\max }=\frac{R_{L}}{R_{S C}+R_{L}} \\
\text { and } \quad & v_{\text {out_max }}=\left(\frac{R_{L}}{R_{S C}+R_{L}}\right)\left(\frac{v_{\text {in }}}{4}\right) .
\end{array}
$$

Of course, other conversion modes can be analyzed by the same method. Table 1 shows the comparison of the SC resistance between the proposed AC-AC converter and the conventional AC-AC converter. As Table 1 shows, the SC resistance of the proposed AC-AC converter is smaller than that of the conventional AC-AC converter. In other words, the proposed AC-AC converter can achieve higher power efficiency than the conventional AC-AC converter.

TABLE I: SC resistance obtained by theoretical analysis

\begin{tabular}{lcc}
\hline \hline & $4 \times$ Step-up & $1 / 4 \times$ Step-down \\
\hline Proposed AC-AC converter & $24 R_{\text {on }}$ & $\frac{3}{2} R_{\text {on }}$ \\
Conventional AC-AC converter & $48 R_{\text {on }}$ & $3 R_{\text {on }}$ \\
\hline \hline
\end{tabular}

\section{Simulation}

In SPICE simulations, the property of the proposed converter is compared with that of the conventional ACAC converter reported in [7]. The SPICE simulations are performed under conditions that $v_{i n}=220 \mathrm{~V} @ 50 \mathrm{~Hz}, C_{1}$ $=\ldots=C_{6}=33 \mu \mathrm{F}, C_{\text {out }}=1 \mathrm{nF}, R_{o n}=0.83 \Omega, T=10 \mu \mathrm{s}$, and $T_{1}=T_{2}=5 \mu \mathrm{s}$, where $C_{\text {out }}$ denotes the output capacitance of the AC-AC converter.

Figure 6 shows the simulated no-load output voltage in the case of step-down conversion and figure 7 shows the simulated no-load output voltage in the case of step-up conversion. In figures 6 and 7, the I/O terminals of the proposed AC-AC converter shown in figure 3 were set to as shown in Table 2. As figures 6 and 7 show, the proposed AC-AC converter can offer not only stepped-down voltages but also stepped-up voltages. Figure 8 shows the simulated root mean square (rms) voltage $V_{r m p}$ as a function of the output power. As figure 8 shows, the proposed AC-AC converter can provide a higher output voltage than the conventional converter. Figure 9 
shows the simulated power efficiency as a function of the output power. As figure 9 shows, the power efficiency of the proposed AC-AC converter is higher than that of the conventional AC-AC converter. Concretely, in the case of the 1/4x step-down conversion, the proposed AC-AC converter can improve power efficiency more than $17 \%$ when the output power is $0.25 \mathrm{~kW}$. In a range from $0.01 \mathrm{~kW}$ to $0.38 \mathrm{~kW}$, the proposed AC-AC converter can achieve more than $80 \%$ efficiency. On the other hand, in the case of the $4 \mathrm{x}$ step-up conversion, the proposed AC-AC converter can improve power efficiency more than $18 \%$ when the output power is $4 \mathrm{~kW}$. In a range from $1.35 \mathrm{~kW}$ to $5.50 \mathrm{~kW}$, the proposed AC-AC converter can achieve more than $80 \%$ efficiency. Of course, the power efficiency of the proposed AC-AC converter depends on the on-resistance of bidirectional switches, clock frequency, capacity values, and so on. Figure 10 shows the input power factor as a function of the output power. As figure 10 shows, the input power factor of the proposed $\mathrm{AC}-\mathrm{AC}$ converter is higher than that of the conventional AC-AC converter. Concretely, in the case of the 1/4x step-down conversion, the proposed AC-AC converter can improve power factor more than 0.51 when the output power is $0.25 \mathrm{~kW}$. The input power factor of the proposed AC-AC converter is more than 0.8 when the output power is higher than $0.25 \mathrm{~kW}$. On the other hand, in the case of the $4 \mathrm{x}$ step-down conversion, the proposed AC-AC converter can improve power factor more than 0.14 when the output power is $4 \mathrm{~kW}$. The input power factor of the proposed AC-AC converter is more than 0.8 when the output power is higher than $2.75 \mathrm{~kW}$.

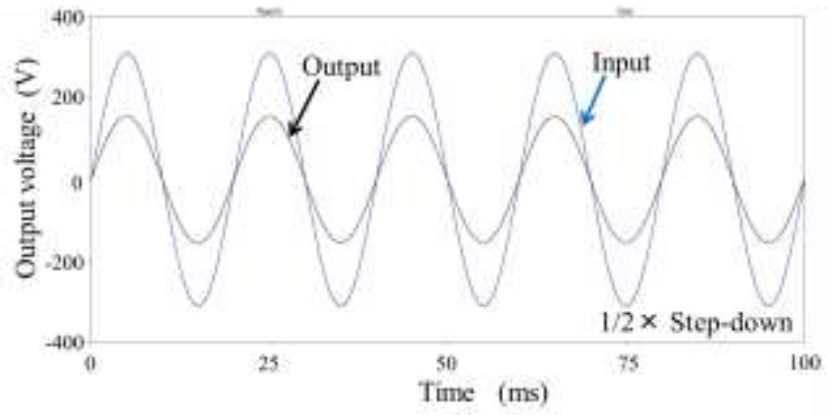

(a)

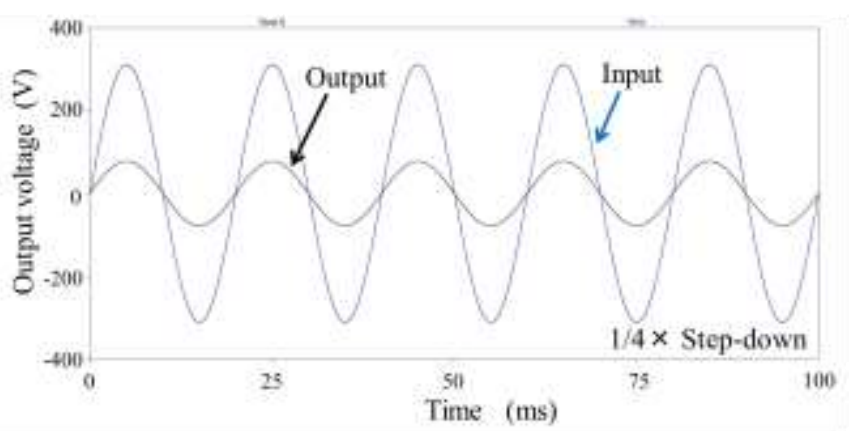

(b)

Fig. 6: Simulated no-load output voltage in the case of step-down conversion: (a) 1/2x step-down and (b) 1/4x step-down.

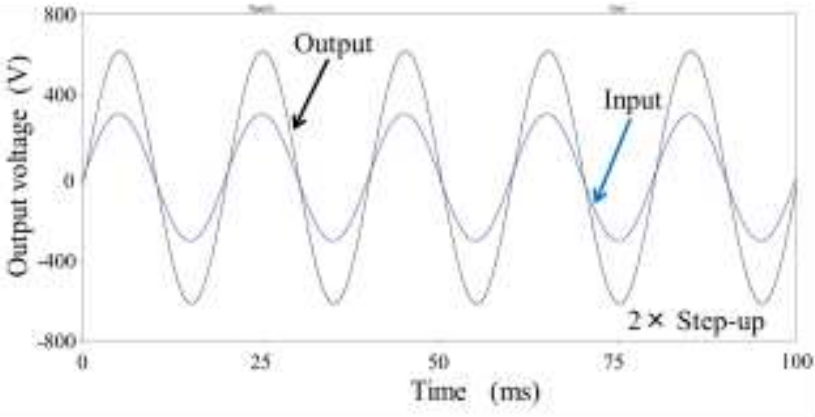

(a)

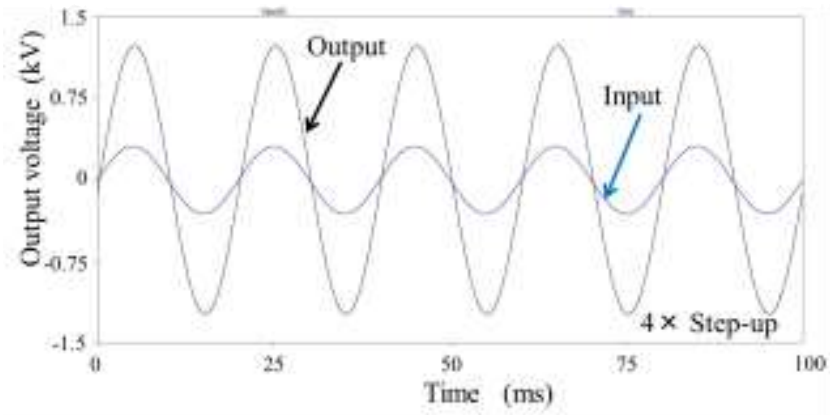

(b)

Fig. 7: Simulated no-load output voltage in the case of step-up conversion: (a) 2x step-up and (b) 4x step-up.

TABLE II: Setting of I/O terminals

\begin{tabular}{lcccc}
\hline \hline & $1 / 2 \times$ Step-up & $1 / 4 \times$ Step-down & $2 \times$ Step-up & $4 \times$ Step-up \\
\hline Input & $v_{\text {in } 2}$ & $v_{\text {in } 4}$ & $v_{\text {in } 1}$ & $v_{\text {in } 1}$ \\
Output & $v_{\text {out } 1}$ & $v_{\text {out } 1}$ & $v_{\text {out } 2}$ & $v_{\text {out } 4}$ \\
\hline \hline
\end{tabular}




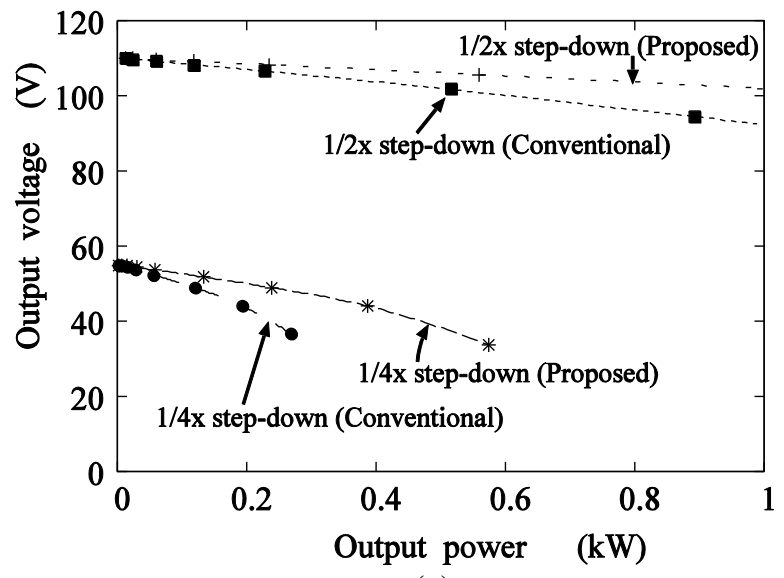

(a)

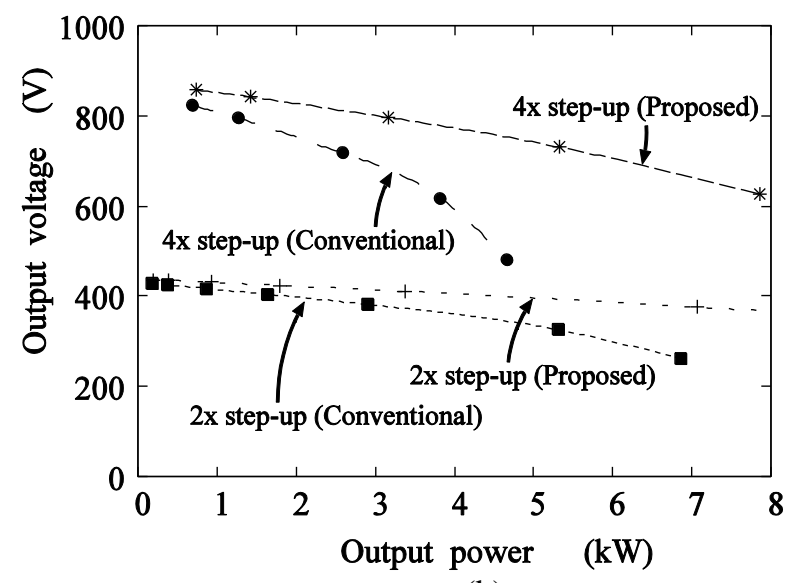

(b)

Fig. 8: Simulated root mean square voltage: (a) Step-down conversion and (b) Step-up conversion.

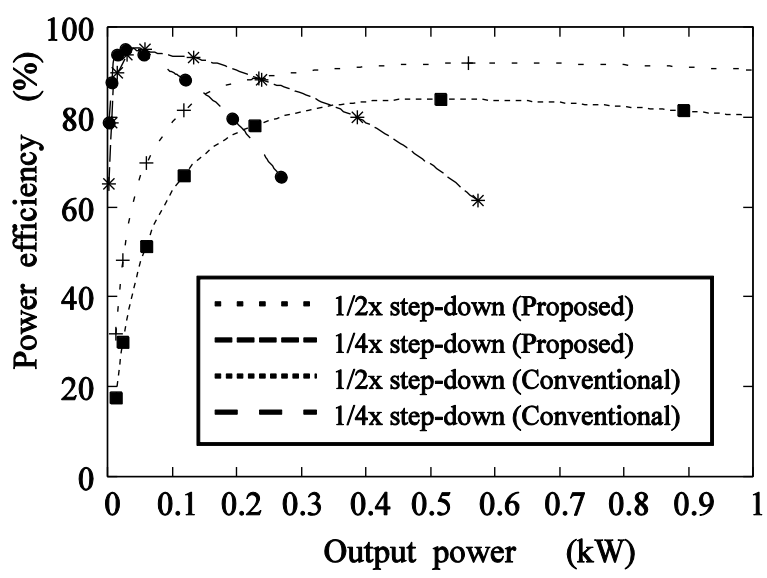

(a)

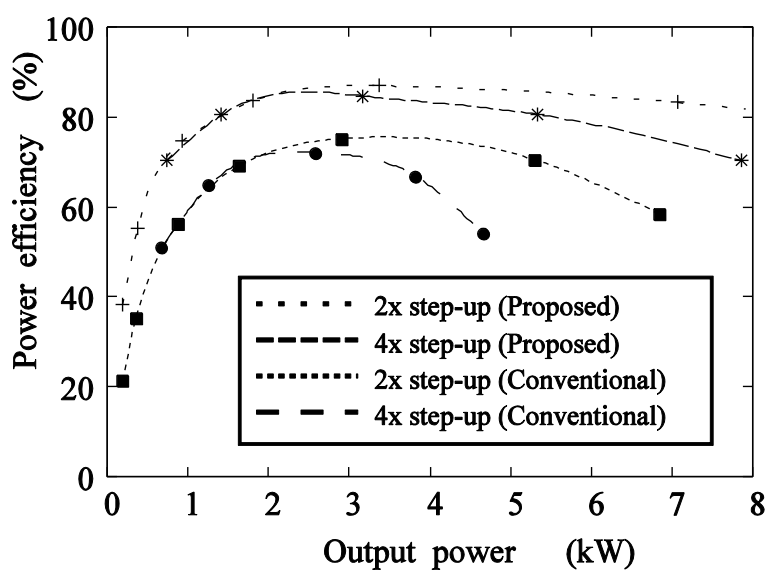

(b)

Fig. 9: Simulated power efficiency: (a) Step-down conversion and (b) Step-up conversion.

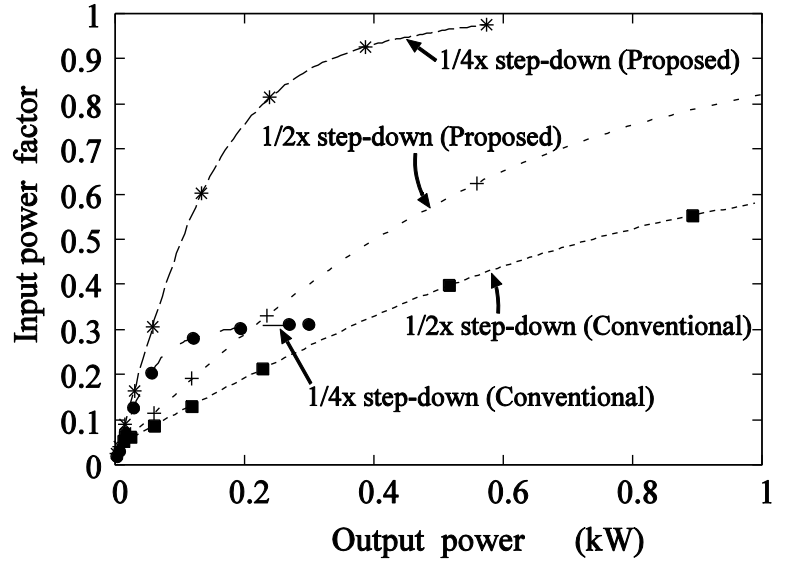

(a)

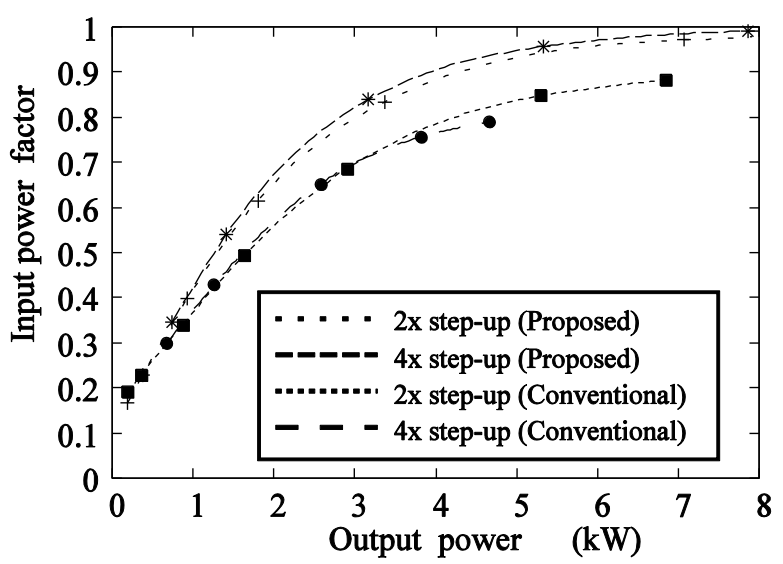

(b)

Fig. 10: Simulated input power factor: (a) Step-down conversion and (b) Step-up conversion.

\section{Conclusion}

A novel switched capacitor (SC) step-up/step-down AC-AC converter has been proposed in this paper. The simulation program with integrated circuit emphasis (SPICE) simulation showed the following results:

1. In the case of the 1/4x step-down conversion, the proposed AC-AC converter improved power efficiency more than $17 \%$ from the conventional $\mathrm{AC}-\mathrm{AC}$ converter when the output power is $0.25 \mathrm{~kW}$. On the other hand, 
in the case of the $4 \mathrm{x}$ step-up conversion, the proposed $\mathrm{AC}-\mathrm{AC}$ converter improved power efficiency more than $18 \%$ when the output power is $4 \mathrm{~kW}$. As these results show, the proposed AC-AC converter can achieve higher power efficiency than the conventional AC-AC converter.

2. In the case of the 1/4x step-down conversion, the proposed AC-AC converter improved input power factor more than 0.51 when the output power is $0.25 \mathrm{~kW}$. On the other hand, in the case of the $4 \mathrm{x}$ step-down conversion, the proposed AC-AC converter improved input power factor more than 0.14 when the output power is $4 \mathrm{~kW}$. As these results show, the proposed AC-AC converter can achieve higher power factor than the conventional ACAC converter.

The experiment concerning the proposed AC-AC converter is left to a future study.

\section{References}

[1] F. Ueno, T. Inoue, I. Oota, and I. Harada, "Realization of a switched-capacitor AC-AC converter," in Proc. 11-th European Conference on Circuit Theory and Design, 1993, pp. 1177-1180.

[2] S. Terada, I. Oota, K. Eguchi, and F. Ueno, "A ring type switched-capacitor (SC) programmable converter with DC or AC input/ DC or AC output," in Proc. 2004 IEEE International Midwest Symposium on Circuits and Systems, 2004, vol.1, pp. I-29-32. http://dx.doi.org/10.1109/mwscas.2004.1353889

[3] K. Eguchi, I. Oota, S. Terada, and T. Inoue, "A design method of switched-capacitor power converters by employing a ring-type power converter," International Journal of Innovative Computing, Information and Control, vol. 5, no.10 (A), pp. 2927-2938, Oct. 2009.

[4] T. B. Lazzarin, R. L. Andersen, G. B. Martins, and I. Barbi, "A $600 \mathrm{~W}$ switched-capacitor ac-ac converter for 220 V/110 V and 110 V/220 V applications," IEEE Trans. on Power Electronics, vol. 27, no.12, pp. 4821-4826, Dec. 2012. http://dx.doi.org/10.1109/TPEL.2012.2203318

[5] T. B. Lazzarin, M. P. Moccelini, and I. Barbi, "Direct buck-type AC/AC converter based on switched-capacitor," in Proc. 2013 Brazilian Power Electronics Conference, 2013, pp. 27-31. http://dx.doi.org/10.1109/COBEP.2013.6785120

[6] R. L. Andersen, T. B. Lazzarin, and I. Barbi, "A 1-kW step-up/step-down switched-capacitor AC-AC converter," IEEE Trans. on Power Electronics, vol. 28, no.7, pp. 3329-3340, July 2013. http://dx.doi.org/10.1109/TPEL.2012.2222674

[7] J. You and C. Hui, "A novel switched-capacitor AC-AC converter with a ratio of 1/4," in Proc. 2014 Int. Conf. on Electrical Machines and Systems, 2014, pp. 3205-3207. http://dx.doi.org/10.1109/ICEMS.2014.7014044

[8] N. Hara, I. Oota, and F. Ueno, "A continuous current switched-capacitor DC-DC converter with fixed-capacitors and a voltage averaging capacitor," in Proc. 1997 International Symposium on Nonlinear Theory and its Applications, 1997, pp. 1209-1212.

[9] K. Eguchi, S. Pongswatd, K. Tirasesth, H. Sasaki, and T. Inoue, "Optimal design of a single-input parallel DC-DC converter designed by switched capacitor techniques," International Journal of Innovative Computing, Information and Control, vol. 6, no.1 (A), pp. 215-227, Jan. 2010. 